\title{
RECENT ADVANCES IN DETECTION AND DIAGNOSIS OF DENTAL CARIES
}

Prachi Mital ${ }^{1}$, Neha Mehta ${ }^{2}$, Aditya Saini ${ }^{3}$, Deepak Raisingani ${ }^{4}$, Medhavi Sharma ${ }^{5}$

\section{HOW TO CITE THIS ARTICLE:}

Prachi Mital, Neha Mehta, Aditya Saini, Deepak Raisingani, Medhavi Sharma."Recent Advances in Detection and Diagnosis of Dental Caries". Journal of Evolution of Medical and Dental Sciences 2014; Vol. 3, Issue 01, January 06; Page: 177-191.

ABSTRACT: Dental caries, a progressive bacterial damage to teeth, is one of the most commondiseases that affects $95 \%$ of the population and is still a major cause of tooth loss.Recent years have seen an increase in research activity surrounding diagnostic methods, particularly in the assessment of early caries lesions. The use of technologies as adjunct to clinical visual examination for caries diagnosis will facilitate preventive care in dentistry to lower treatment cost as well as reduce the cost and time for testing potential anticaries agents. This article describes the various technologies available to aid the dental practitioners in detecting and diagnosis ofdental caries at the earliest stage of its formation, assessing the activities of the detected carious lesion, and quantitatively or qualitatively monitoring of the lesion over time.

KEYWORDS: Dental caries, Diagnosis, Detection.

INTRODUCTION: "Any dentist who cannot find any cavity of decay on any tooth with the explorer and mouth glass needs a competent assistant to make his examinations for him, and not a roentgenray machine."

C. Edmund Kells before the American Dental Association November 10-14, 19241.

Dental caries is an infectious microbiologic disease of the teeth that results in localized dissolution and destruction of calcified tissues. ${ }^{2}$ Development of caries require interaction between plaque microorganism, susceptible tooth, substrate and time. The infection results in loss of tooth mineralsthat begins on the outer surface of the tooth and can progress through the dentin to the pulp, ultimately compromising the vitality of the tooth. There are number of risk and modifying factors (Table 1) which affectthe mineral equilibrium in one direction or another, i.e. towards remineralisation or demineralisation. ${ }^{3}$ There has been remarkable progress in the reduction of dental caries over the past 30 years. Changes have been observed not only in the prevalence of dental caries, but also in the distribution and pattern of the disease in the population. Specifically, it has been observed that the relative distribution of dental caries on tooth surfaces has changed, and the rate of lesion progression through the teeth is relatively slow for most people. The use of fluoride in public water supplies, in toothpaste, and in professional dental products, improved oral hygiene, and increased access to dental care have played major roles in this dramatic improvement. Nevertheless, dental caries remains a significant problem. Finding an accurate method for detecting and diagnosing any disease has been the goal of the healing arts since the time of Socrates.

Early diagnosis of the caries lesion is important because the carious process can be modified by preventive treatment so that the lesion does not progress. If the caries disease can be diagnosed at an initial stage (e.g. white spot lesion) the balance can be tipped in favor of arrestment of the process by modifying diet, improving plaque control, and appropriate use of fluoride. ${ }^{4}$ Using noninvasive quantitative diagnostic methods it should be possible to detect lesions at an initial stage and 
subsequently monitor lesion changes over time during which preventive measures could be introduced.

Caries detection methods: Caries detection methods should be capable of detecting lesions at an early stage, when progression can be arrested or reserved, avoiding premature tooth treatment by restorations.

\section{I) Conventional Methods:}

Visual-tactile examination- Visual examination has been widely used in dental clinics for detecting carious lesions on all surfaces. This method is based on the use of a dental mirror, a sharp probe and a 3-in-1syringe and requires good lighting and a clean/dry tooth surface. Decision (absence or presence of a lesion) is based on subjective interpretation of integrity, texture, translucency/opacity, location and color.5, 6 Visual examination has been shown to have a high specificity but low sensitivity and reproducibility ${ }^{7}$ They cannot detect caries lesions until a relatively advanced stage, involving one-third or more of the thickness.

The International Caries Detection \& Assessment System (ICDAS) - the systemwas developed and introduced by an international group of researchers (cariologists and epidemiologists) to provide clinicians, epidemiologists, and researchers with an evidence-based system for caries detection (Pitts, 20048). This method was devised based on the principle that the visual examination should be carried out on clean, plaque-free teeth, with carefully drying of the lesion / surface to identify early lesions. According to this system, the replacement of the traditional explorers and sharp probes with a ball-ended periodontal probe would avoid traumatic and iatrogenic defects on incipient lesions 9,10

ICDAS is a two-digit identification system. Initially, the status of the surface is described as unrestored, sealed, restored or crowned. After that, a second code is attributed to identify six stages of caries extension, varying from initial changes visible in enamel to frank cavitation in dentine ${ }^{9}$, ${ }^{10}$ (Table 2)

II) Novel diagnostic systems: Novel diagnostic systems are based upon the measurement of a physical signal-these are surrogate measures of the caries process. Examples of the physical signals that can be used in this way include X-rays, visible light;laser light, electronic current, ultrasound, and possibly surface roughness. For a caries detection device to function, it must be capable of initiating and receiving the signal as well as being able to interpret the strength of the signal in a meaningful way. Table 3 demonstrates the physical principles and the detection systems that have taken advantage of them. ${ }^{11}$

\section{A) Radiographic techniques}

i) Conventional radiography: Radiology described in 1923 as "the most revolutionary aid in dental diagnosis which has come into general application during the present decade," The use of a bitewing radiography as an adjunct to the clinical examination could permit more sensitive detection of proximal and occlusal caries lesions in dentin and a better estimation of the lesion depth than the visual inspection performed alone. Moreover, the monitoring of caries lesions could be more reliable 
and accurate than using the conventional clinical examination alone.Bitewing projection is the most appropriate radiographic technique for caries detection. This technique requires a film-holder with a wing for the patient to bite.

ii) Digital radiography: Digital radiography has offered the potential to increase the diagnostic yield of dental radiographs. It has manifested itself in subtraction radiography. A digital radiograph is comprised of a number of pixels. Each pixel carries a value between 0 and 255, with 0 beingblack and 255 being white.The values in between represent shades of grey, and it can be quickly appreciated that a digital radiograph, with a potential of 256 grey levels has significantly lower resolution than a conventionalradiograph that contain millions of grey levels. ${ }^{12}$

Sensitivities and specificities of digital radiographs being significantly lower than those of regular radiographs when assessing small proximal lesions. However, digital radiographs offer the potential ofimage enhancement by applying a range of algorithms, some of which enhance the white end of the grey scale (such as Rayleigh and hyperbolic logarithmic probability) and others the blackend (hyperbolic cube root function).

Digital radiographs offer a decrease in radiographic dose and thus offer additionalbenefits than diagnostic yield. Digital images can also be archived and replicated with ease.

iii) Digital subtraction radiography: Digital subtraction radiography (DSR) is a more advanced image analysis tools. Thismethod allowsto distinguish small differences between subsequent radiographs that otherwise would have remained unobserved because of over projection of anatomical structures or differences in density that are too small to be recognized by the human eye. Digital subtraction radiography has been used in the assessment of the progression, arrest, or regression of caries lesions. The basic premise of subtraction radiology is that two radiographs of the same object can be compared using their pixel values. The value of the pixels from the first object is subtracted from the second image. If there is no change, the resultant pixel will be scored 0; any value that is not 0 must be attributable to either the onset or progression of demineralisation, or regression. When there is caries regression, the outcome will be a value above zero (increase in pixel values). In case of caries regression, the result is opposite and the outcome will be a value below zero (decrease in pixel values). ${ }^{13}$ Subtraction images therefore emphasise this change and the sensitivity is increased. ${ }^{14,15}$

\section{B) Enhanced visual techniques}

i) Fibre optic transillumination (FOTI):visual inspection of caries is based upon the phenomenon of light scattering. Sound enamel is comprised of modified hydroxyapatite crystals that are densely packed, producing an almosttransparent structure.

PRINCIPLE OF FOTI:It works because of the different index of light transmission for decay, sound tooth structure and healthy periodontium. Since the carious tooth structure has a lower index of light transmission than sound tooth structure, an area of decay shows up as a darkened shadow that follows the spread of decay along the path of dentinal tubules.Fibre optic transillumination uses high intensity white light that is presented through a small aperture in the form of a dental handpiece. The tip is $0.5 \mathrm{~mm}$;light source is by a 150 watt halogen lamp set at maximum intensity. The probe is 
applied perpendicular to the buccal and lingual surfaces and its position and angulation varied to obtain maximum light scattering through the lesion. The decrease of transmission is interpreted by the observer, traditionally as an ordinary rating scale.

Shadow depth scaleScore 0 = sound

Score 1 = shadow in enamel

Score 2 = shadow in dentine

It is simple, noninvasive, painless procedure that can be used repeatedly with no risk to the patient. It can be used for thedetection of caries on all surfaces; and is particularly useful at proximal lesions. The research around FOTI is somewhat polarised, with a recent review finding a mean sensitivity of only 14 and a specificity of 95 when considering occlusal dentine lesions, and 4 and $100 \%$ for proximal lesions. There are some limitations of FOTI, the system is subjective rather than objective. There is no continuous data outputted.It is not possible to record what is seen in the form of an image.(Fig -01a, 01b)

ii) Digital Imaging Fiber-Optic TransIllumination (DIFOTI): Developed by Schneidermann et al, Department of Oral Pathology, Radiology and Diagnostic Sciences, New Jersy Dental School, University of Medicine and Dentistry of NewJersey. Images of teeth are obtained using visible light via fiber optic transillumination. The images are acquired by a digital electronic CCD camera, therefore DIFOTI may eliminate or reduce intra- and inter-observer variation. Light propogates from the optic fiber throughthe tooth to a non-illuminated surface (usually the opposite surface). Thus, DIFOTI images can be acquired in repeatable fashion by maintaining adjustment of a number of imaging control parameters. Then the acquired information is sent to a computer for analysis with dedicated algorithms, which produce digital images that can be viewed by the dentist and patient in real time or stored for later assessment. In addition, this system can use digital image processing methods to enhance contrast between sound and carious tissues and to quantify features of incipient, frank and secondary caries lesions on occlusal, approximal and smooth surfaces. It can also be used to detect other changes in coronal tooth anatomy, such as tooth fractures and fluorosis. DIFOTI presents higher sensitivity in detection early lesions when compared to the radiographic examination and has potential for quantitative monitoring of selected lesions over a period of time ${ }^{16}$, ${ }^{17}$ However approximal lesions can be picked up by using DIFOTI only by careful angulation. It does not measure lesion depth since the resulting image is that of a surface or what is near the surface. There is inability to quantify lesion progression, even though images can be compared over time.With the greater sensitivity and somewhat lower specificity there is real possibility of over diagnosis. (Fig -.02)

\section{C) Fluorescent techniques}

i) Quantitative Light-Induced Fluorescence (QLF) Quantitative light-induced fluorescence (QLF) is a visible light system that offers the opportunity to detect early caries and then longitudinally monitor their progression or regression. Using two forms of fluorescent detection (green and red) it may also be able to determine if a lesion is active or not, and predict the likely progression of any given lesion. Fluorescenceis a phenomenon by which an object is excited by a particular wavelength of light and the fluorescent (reflected) light is of a larger wavelength. When the excitation light is in 
the visible spectrum, the fluorescence will be of a different color. In the case of the QLF the visible light has a wavelength (l) of $370 \mathrm{~nm}$, which is in the blue region of the spectrum. The resultant autofluorescence of human enamel is then detected by filtering out the excitation light using a bandpass filter at l $>540 \mathrm{~nm}$ by a small intra-oral camera. This produces an image that is comprised of only green and red channels (the blue having been filtered out) and the predominant color of the enamel is green.18,19 Demineralization of enamel results in a reduction of this auto-fluorescence. This loss can be quantified using proprietary software and has been shown to correlate well with actual mineral loss; $r=0.73-0.86 . .^{20}$

Principle: Autofluorescence of the tooth alters as the mineral content of the dental hard tissue changes. Increased porosity due to a subsurface enamel lesion scatters the light either as it enters the tooth or as the fluorescence is emitted, resulting in a loss of its natural fluorescence. The changes in enamel fluorescence can be detected and measured when the tooth is illuminated by violet-blue light (wavelengths 290-450 $\mathrm{nm}$, average $380 \mathrm{~nm}$ ) from a camera hand piece, following image capturing using a camera fitted with a yellow $520 \mathrm{~nm}$ high pass filter. The QLF method can also measure and quantify the red fluorescence (RF) from microorganisms in plaque.

The QLF equipment is comprised of a light box containing a xenon bulb and a hand piece, similar in appearance to an intraoral camera, light is passed to the hand piece via a liquid light guide and the hand piece contains the band pass filter.21 Live images are displayed via a computer and accompanying software enables patient's details to be entered and individual images of the teeth of interest to be captured and stored. QLF can image all tooth surfaces except inter- proximally. Once an image of a tooth has been captured, the next stage is to analyze any lesions and produce a quantitative assessment of the demineralization status of the tooth. (Fig -03a \& 03b)

\section{D) Laser-Induced Fluorescence}

i) DIAGNODent: DIAGNOdent contains a laser diode (655 $\mathrm{nm}$, modulated, $1 \mathrm{~mW}$ peak power) as the excitation light source, and a photo diode combined with a long pass filter (transmission > $680 \mathrm{~nm}$ ) as the detector. The excitation light is transmitted by an optical fiber to the tooth, and a bundle of 9 fibers arranged concentrically around it serves for detection. The long pass filter absorbs the backscattered excitation and other short wavelength light and transmits the longer wavelength fluorescence radiation. To eliminate the long wavelength ambient light also passing through the filter, the laser diode is modulated, and only light showing the same modulation characteristic is registered. Thus, the digital display shows quantitatively the detected fluorescence intensity (in units related to a calibration standard).

The emitted light reaches the dental tissues through a flexible tip. As the mature enamel is more transparent, this light passes through this tissue without being deflected. In contact with affected enamel, this light will be diffracted and dispersed. The latter is able to excite either the hard dental tissue, resulting in the tissue autofluorescence, or fluorophores present in the caries lesions. These fluorophores derived from the products of the bacterial metabolism and has been identified as porphyrins ${ }^{22}$. The emitted fluorescence by the porphyrins is collected by nine concentric fibers and translated into numeric values, which can vary from 0 to 99. Two optical tips are available: tip A for occlusal surfaces, and tip B for smooth surfaces. This device has shown good results in the detection 
of occlusal caries, however, it might not be used as the only method for treatment decision-making process $^{23,24}$ (Fig -04)

ii) Fluorescence camera (VistaProof): Fluorescence camera (VistaProof) is based on the lightinduced fluorescence phenomenon. (Dürr Dental, Bietigheim-Bissingen, Germany) that is based on six blue GaN-LEDs emitting a 405-nm light. With this camera it is possible to digitize the video signal from the dental surface during fluorescence emission using a CCD sensor (charge coupled device). On these images, it is possible to see different areas of the dental surface that fluoresce in green (sound dental tissue) and in red (carious dental tissue) ${ }^{25}$. DBSWIN software is used to analyze the images and translate into values the intensity ratio of the red and green fluorescence. The software highlights the lesions and classifies them in a scale from 0 to 5 , giving a treatment orientation in the first evaluation: monitoring, remineralization or invasive treatment ${ }^{26}$.

iii) LED technology (Midwest Caries I.D.): Recently, a device based onLED technology - Midwest Caries I.D. - (DENTSPLY Professional, York, PA, USA) is developed for caries detection. The handheld device emits a soft light emitting diode (LED) between $635 \mathrm{~nm}$ and $880 \mathrm{~nm}$ and analyzes the reflectance and refraction of the emitted light from the tooth surface, which is captured by fiber optics and is converted to electrical signals for analysis. The microprocessor of the device contains a computer-based algorithm that identifies the different optical signature (changes in optical translucency and opacity) between healthy and demineralized tooth ${ }^{27}$

E) Detection systems based on electrical current measurement: Every material possesses its own electrical signature; i.e. when a current is passed through the substance the properties of the material dictate the degree to which that current is conducted. Conditions in which the material is storedor physical changes to the structure of the material will have an effect on this conductance.

The concentration of fluids and electrolytes contained within Biological materials largely govern their conductivity. ${ }^{28}$ For example, dentine is more conductive than enamel. In dental systems, there is generally a probe, from which the current is passed, a substrate, typically the tooth, and a contraelectrode, usually a metal bar held in the patient's hand. Measurements can be taken either from enamel or exposed dentinesurfaces. ${ }^{29}$ In its simplest form, caries can be described as a process resulting in an increase in porosity of the tissue (enamel or dentine). This increased porosity results in a higher fluid content than sound tissue and this difference can be detected by electrical measurement by decreased electrical resistance or impedance. Based on the differences in the electrical conductance of carious and sound enamel two instruments were developed and tested in 1980's.

i) Vangaurd electronic caries detectormanufactured by Massachusetts Manufacturing Corp., Cambridge, Mass, USA. Electrical conductivity is expressed numerically on a scale from 0 to 9 . The machine displayed a frowning face that indicated extensive demineralization or the smiling face that indicated a sound site. This device is no longer available commercially.

ii) Caries meter L. manufactured by two companies-GC international corp, leven, belgium and Onuki dental corp, Itd, japan. Each measurement site is moistened with saliva to ensure proper 
contact between the electrode and the tooth. The Caries Meter L uses colored lights to indicate caries extent.

- Green-sound

- Yellow-enamel caries

- Orange-dentinal caries

- Red-caries reaching the pulp

The system is painless, and safe for the patient. X-rays can be avoided and the diagnosis/checkup offers the patient insight into his or her oral hygiene.The measurement system fits in (with) the existing routine and neither special knowledge nor extra time is required. It may become part of the checkup that is performed by the assistant or dental hygienist.

Electronic caries monitor (ECM) -The ECM device employs a single, fixed-frequency alternating current which attempts to measure the 'bulk resistance' of tooth tissue ${ }^{30}$ This can be undertaken at either a site or surface level. When measuring the electrical properties of a particular site on a tooth, the ECM probe is directly applied to the site, typically a fissure, and the site measured. During the $5 \mathrm{~s}$ measurement cycle, compressed air is expressed from the tip of the probe and this results in a collection of data over the measurement period, described as a drying profile that can provide useful information for characterizing the lesion. It is generally accepted that the increase in porosity associated with caries is responsible for the mechanism of action for ECM,31 There are number of physical factors that will affect ECM results. These include the temperature of the tooth, 31 the thickness of the tissue, ${ }^{32}$ the hydration of the material (i.e. one should not dry the teeth prior to use) and the surface area. ${ }^{30}$ The ECM readings may range between -0.70 and 13.20 indicating increased conductance.(Fig - 05).

\section{Interpretation of values}

-1.00 to $3.00=$ sound enamel or incipient stage of caries

3.01 to $6.00=$ caries upto the DEJ (enamel caries)

6.01 to $8.00=$ dentinal caries

8.01 to $13.00=$ extending half of dentine thickness

F) Ultrasound techniques: The principle behind the technique is that sound waves can pass through gases, liquids and solids and the boundaries between them.Images of tissues can be acquired by collecting the reflected sound waves. In order for sound waves to reach the tooth they must pass first through a coupling mechanism, usually water and glycerine ${ }^{33}$

The pulse echo ultrasonic waves mean that any impulse generated in the transducer is transmitted into a medium (tooth) and then reflected back to the transducer if it strikes at any discontinuity. Sound and demineralized can be differentiated from their echo position on the CRT.

The Ultrasonic System: Ultrasonic system is composed of a transducer (probe) \& an ultrasonic precision thickness gauge.The contact transducer is of the right angle type with a $1.5 \mathrm{~mm}$ tip contact diameter, $11 \mathrm{MHz}$ nominal center frequency with a removable plexiglass delay tip. The delay line tip of the contact transducer is at a right angle so that it could be inserted or used in restricted height areas. These high frequency focused delay line transducers are compatible with any ultrasonic 
instrument capable of displaying a return echo at depths as minute as 0.010 inches. The useful range is from 0.010 to 0.25 inches approximately.

Chemical Agents For Detection Of Dental Caries: Caries indicator dyes are non-specific protein dyes that stain the organic matrix of less mineralized dentin, including normal circumpulpal dentin and sound dentin in the area of the amelo-dentinal junction. It is a diagnostic aid for detecting occlusal caries.The dye is purported to stain only infected tissue and is advocated for a "painless" caries removal technique without local anesthetic. The technique is laborious, as it isguided by staining, involvesmultiple dye application-and-removal repetitions and requires the use of a slowspeed bur.

Chemomechanical removal of caries(CARISOLV): Involves chemical softening of carious dentine followed by its removal by gentle excavation.Carisolv was developed in January 1998in Sweden. It is gel containing Glutamicacid, Leucine, Lysine, Sodium chloride, Erythrosine, Carboxymethylcellulose, Water, sodium hydroxide, and a transparent fluid containing $0.5 \%$ sodium hypochlorite.

CONCLUSION: The pattern of dental caries is changing, with an increasing incidence in occlusal surfaces. This shift has rendered traditional detection systems, particularly bitewing radiographs less useful in the diagnostic protocols of clinicians. High concentration fluoride varnishes have been demonstrated to arrest the progression of early lesions, but often traditional methods of detection are too insensitive to permitthe most efficacious use of these products. Although currently there is no single diagnostic method on the horizon that can reliably detect pre-cavitated carious lesions on all tooth surfaces, the prospects look favorable that, with continued research, laser fluorescence, quantitative light induced fluorescence, electrical conductivity measurements, direct digital radiography, and digital fiberoptic transillumination, will provide the high degree of sensitivity and specificity needed to detect early dental caries. Because "the ultimate outcome of the caries diagnostic process in clinical practice is the decision concerning the need for treatment", the development of newer methodologies of caries detection are critical to reduce the possibility of an incorrect diagnosis, which will ultimately lead to an incorrect treatment decision. Without newer methods of caries detection, people who are at low risk for disease progression may receive needless treatment, whereas other high-risk groups may be overlooked, resulting in an undue waste of manpower, time, and health care cost.

\section{REFERENCES:}

1. Raper HR. Practical clinical preventive dentistry based upon periodic roentgen ray examinations. J Am Dent Assoc 1925; 112(9):1084-100.

2. Theodore M.R., Harald O.H, Edward J. S Sturdevant's Art and Science of operative dentistry $5^{\text {th }}$ Edition Elsevier publication, Chapter 3: Cariology: The lesions, etiology, prevention and control; pg 65 - 134

3. Holt RD. Advances in dental public health. Primary Dental Care 2001; 8(3):99-102.

4. Michele Baffi Diniz, Jonas de Almeida Rodrigues and Adrian Lussi Traditional and Novel Caries Detection Methods: uploaded 3rd April 2012 by Jonas Almeida Rodrigues 
5. Ekstrand, K.R.; Ricketts, D.N. \& Kidd, E.A. Reproducibility and accuracy of three methods for assessment of demineralization depth on the occlusal surface: an in vitro examination. Caries Research. 1997; Vol.31, No.3, pp. 224-231, ISSN 0008-6568

6. Nyvad, B.; Machiulskiene, V. \& Baelum, V. Reliability of a new caries diagnostic system differentiating between active and inactive caries lesions. Caries Research. 1999;Vol.33, No.4, (July-August), pp. 252-260, ISSN 0002-8177

7. Bader, J.D.; Shugars, D.A. \& Bonito, A.J. Systematic reviews of selected dental caries diagnosis and management methods. Journal of Dental Education. 2001;Vol.65, No.10, (October), pp. 960-968, ISSN 0022-0337

8. Pitts, N. "ICDAS" -- an international system for caries detection and assessment being developed to facilitate caries epidemiology, research and appropriated clinical management. Community Dental Health. 2004;Vol.21, No.3, (September), pp. 193-198, ISSN 0256-539X

9. Ekstrand, K.R.; Martignon, S.; Ricketts, D.J. \& Qvist, V. Detection and activity assessment of primary coronal caries lesions: a methodologic study. Operative Dentistry. 2007;Vol.32, No.3, (May-June), pp. 225-235, ISSN 0361-7734

10. Ismail, A.I.; Sohn, W.; Tellez, M.; Amaya, A.; Sen, A.; Hasson, H. \& Pitts, N.B. The International Caries Detection and Assessment System (ICDAS): an integrated system for measuring dental caries. Community Dentistry and Oral Epidemiology. 2007;Vol.35, No.3, (June), pp. 170-178, ISSN 0301-5661

11. Verdonschot EH, Angmar-Mansson B. Advanced methods of caries diagnosis and quantification. In: Fejerskov O, Kidd E, editors. Dental Caries. The disease and its clinical management. Oxford: Blackwell Munksgaard; 2003.

12. Iain A. Pretty Caries detection and diagnosis: Novel technologies; journal of dentistry 34 (2006) $727-739$

13. Hekmatian, E.; Sharif, S. \& Khodaian, N. Literature review: digital subtraction radiography in dentistry. Dental Research Journal. 2005;Vol.2, No.2, pp. 1-8, ISSN 1735-3327

14. van der Stelt, P.F. Better imaging: the advantages of digital radiography. Journal of the American Dental Association. 2008;Vol.139, No. Suppl, (June), pp. 7S-13S, ISSN 0002-8177

15. Wenzel, A. Bitewing and digital bitewing radiography for detection of caries lesions. Journal of Dental Research. 2004;Vol.83, No. Spec No C, pp. C72-C75, ISSN 0022-0345

16. Bin-Shuwaish, M.; Dennison, J.B.; Yaman, P. \& Neiva, G. Estimation of clinical axial extension of class II caries lesions with ultraspeed and digital radiographs: an invivo study. Operative Dentistry. 2008;Vol.33, No.6, (November-December), pp. 613-621, ISSN 0361-7734

17. Young, D.A. \& Featherstone, J.D. Digital imaging fiber-optic trans-illumination, Fspeed radiographic film and depth of approximal lesions. Journal of the American Dental Association. 2005; Vol.136, No.12(December), pp. 1682-1687, ISSN 0002-8177

18. de Josselin de Jong E, Sundstrom F, Westerling H, Tranaeus S, ten Bosch JJ, Angmar-Mansson B. A new method for in vivo quantification of changes in initial enamel caries with laser fluorescence. Caries Research 1995;29(1):2-7.

19. Ando M, Hall AF, Eckert GJ, Schemehorn BR, Analoui M, Stookey GK. Relative ability of laser fluorescence techniques to quantitate early mineral loss in vitro. Caries Research 1997;31(2):125-31. 
20. van der Veen MH, de Josselin de Jong E. Application of quantitative light-induced fluorescence for assessing early caries lesions. Monography in Oral Science 2000;17: 144-62.

21. Angmar-Mansson B, ten Bosch JJ. Quantitative light-induced fluorescence (QLF): a method for assessment of incipient caries lesions. Dentomaxillofac Radiology 2001;30(6):298-307.

22. Hibst, R.; Paulus, R. \& Lussi, A. A detection of occlusal caries by laser fluorescence: basic and clinical investigations. Medical Laser Application. 2001;Vol.16, No.3, (June), pp. 295-13, ISSN 1615-1615

23. Bader, J.D. \& Shugars, D.A. The evidence supporting alternative management strategies for early occlusal caries and suspected occlusal dentinal caries. Journal of Evidence-Based Dental Practice. 2006;Vol.6, No.1, (March), pp. 91-100, ISSN 1532-3382

24. Rodrigues, J.A.; Hug, I.; Diniz, M.B. \& Lussi, A. Performance of fluorescence methods, radiographic examination and ICDAS II on occlusal surfaces in vitro.Caries Research. 2008;Vol.42, No.4, (July), pp. 297-304, ISSN 0002-8177

25. Thoms, M. Detection of intraoral lesions using a fluorescence camera. Proceedings of SPIE Lasers in Dentistry XII. 2006;Vol.6137, No.5, pp. 1-7, ISSN 0002-8177

26. Rodrigues, J.A.; Hug, I.; Neuhaus, K.W. \& Lussi, A. Light-emitting diode and laser fluorescencebased devices in detecting occlusal caries. Journal of Biomedical Optics. 2011;Vol.16, No.10, pp. 107003-1-107003-5, ISSN 1083-3668

27. Strassler, H.E. \&Sensi, L.G. Technology-enhanced caries detection and diagnosis. Compendium of Continuing Education in Dentistry. 2008;Vol.29, No.8, (October), pp. 464-465, 468, 470 passim, ISSN 1548-8578

28. Ekstrand KR, Ricketts DN, Kidd EA, Qvist V, Schou S.Detection, diagnosing, monitoring and logical treatment of occlusal caries in relation to lesion activity and severity: an in vivo examination with histological validation. Caries Research 1998;32(4):247-54.

29. Verdonschot EH, Rondel P, Huysmans MC. Validity of electrical conductance measurements in evaluating the marginal integrity of sealant restorations. Caries Research 1995;29(2):100-6.

30. Longbottom C, Huysmans MC. Electrical measurements for use in caries clinical trials. Journal of Dental Research. 2004:83.Spec no. C:C 76-9.

31. Huysmans MC, Longbottom C, Christie AM, Bruce PG, Shellis RP. Temperature dependence of the electrical resistance of sound and carious teeth. Journal of Dental Research 2000;79(7):1464-8.

32. Wang J, Sakuma S, Yoshihara A, Kobayashi S, Miyazaki H. An evaluation and comparison of visual inspection. Electrical caries monitor and caries detector dye methods in detecting early occlusal caries in vitro study. Journal of Dental Health 2000;50:223-30.

33. Hall A, Girkin JM. A review of potential new diagnostic modalities for caries lesions. Journal of Dental Research 2004:83. Spec no. C:C89-94.46. Fried D, Featherstone JD, Darling 


\section{REVIEW ARTICLE}

\section{Table 1: Risk and modifying factors for caries}

\section{Primary risk factors}

\section{Saliva}

- Abilityof minor salivary glands to produce saliva

- Consistency of unstimulated (resting) saliva

- $\mathrm{pH}$ of unstimulated saliva

- Stimulated salivary flow rate

- Buffering capacity of stimulated saliva

\section{Diet}

- Number of sugar exposures per day

- Number of acid exposures per day

\section{Fluoride}

- Past and current exposure

\section{Oral biofilm}

- Differential staining

- Composition

- Activity

\section{Modifying factors}

- Past and current dental status

- Past and current medical status

- Compliance with oral hygiene and dietary advice

- Lifestyle

- Socioeconomic status

\begin{tabular}{|c|l|}
\hline Code & \multicolumn{1}{|c|}{ Description } \\
\hline 0 & Sound tooth surface \\
\hline 1 & First visual change in enamel seen only after prolonged drying \\
\hline 2 & Distinct visual change in enamel \\
\hline 3 & Localized enamel breakdown in opaque or discolored enamel, no dentin visible \\
\hline 4 & Dentinal shadow (not cavitated into dentin) \\
\hline 5 & Distinct cavity with visible dentin \\
\hline 6 & Extensive distinct cavity with visible dentin \\
\hline & Table 2: ICDAS II caries severity codes \\
\hline
\end{tabular}




\section{REVIEW ARTICLE}

\begin{tabular}{|c|c|}
\hline Physical principle & Application in caries detection \\
\hline $\mathrm{X}$-rays & $\begin{array}{l}\text { Digital subtraction radiography } \\
\text { Digital image enhancement }\end{array}$ \\
\hline Visible light & $\begin{array}{l}\text { Fibre optic transillumination (FOTI) } \\
\text { Quantitative light-induced fluorescence(QLF) } \\
\text { Digital image fibre optictransillumination(DiFOTI) }\end{array}$ \\
\hline Laser light & Laser fluorescence measurement (DiagnoDent) \\
\hline Electrical current & $\begin{array}{l}\text { Electrical conductance measurement (ECM) } \\
\text { Electrical impedance measurement }\end{array}$ \\
\hline Ultrasound & Ultrasonic caries detector \\
\hline
\end{tabular}

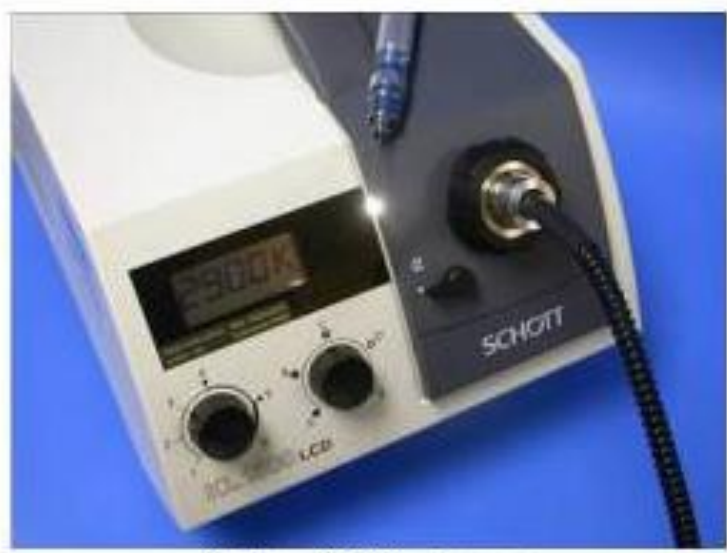

Fig la: FOTI equipment

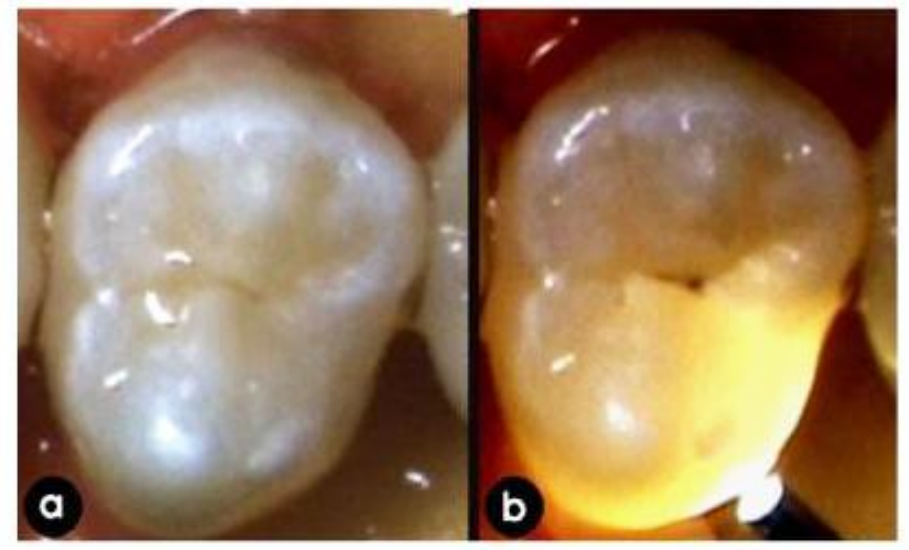

Fig 1(b): Example of FOTI on a tooth. (a) Normal clinical vision, (b) with FOTI 


\section{REVIEW ARTICLE}

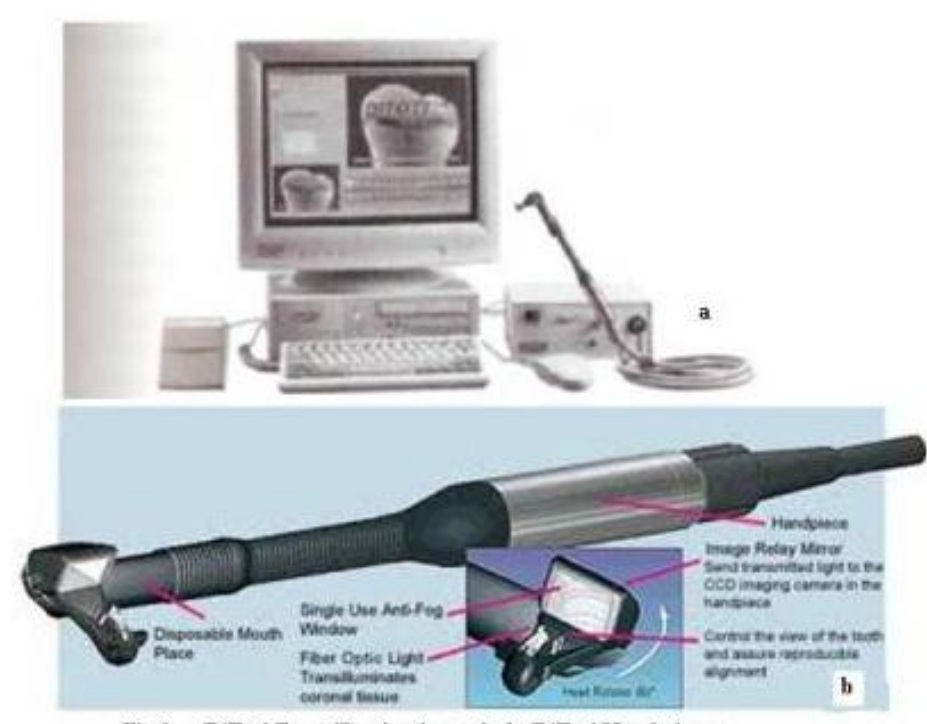

Fig 2 a: DiFoti Transillumination unit, b: DiFoti Hand piece

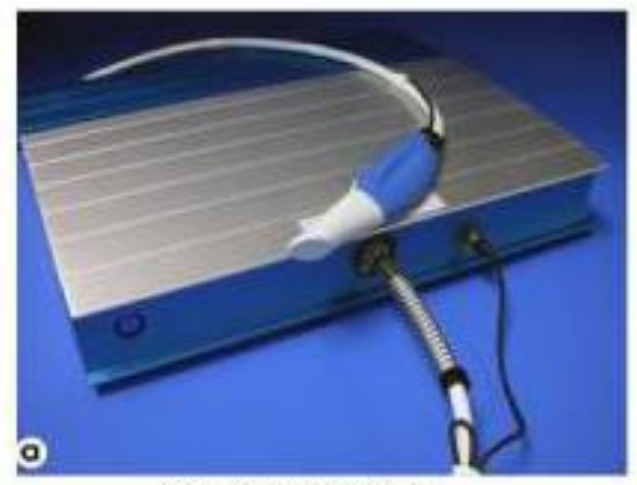

Fig - 03 a: QLF Unit

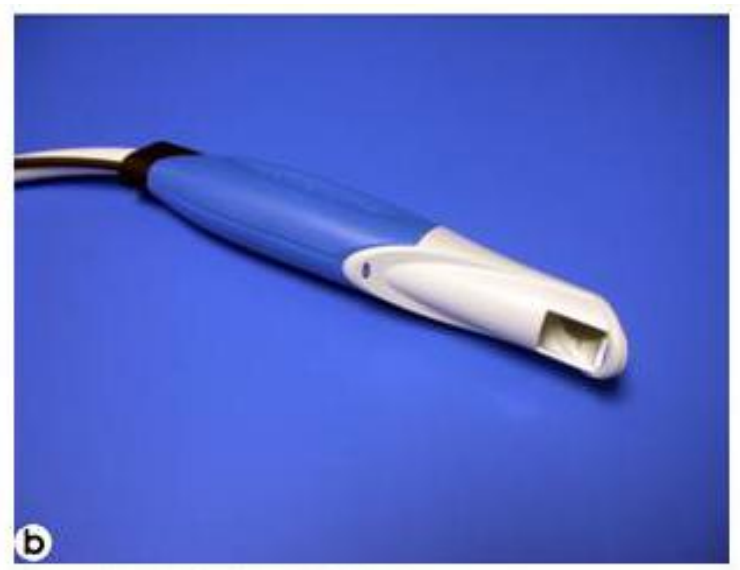

Fig - 03 b: QLF hand piece 


\section{REVIEW ARTICLE}

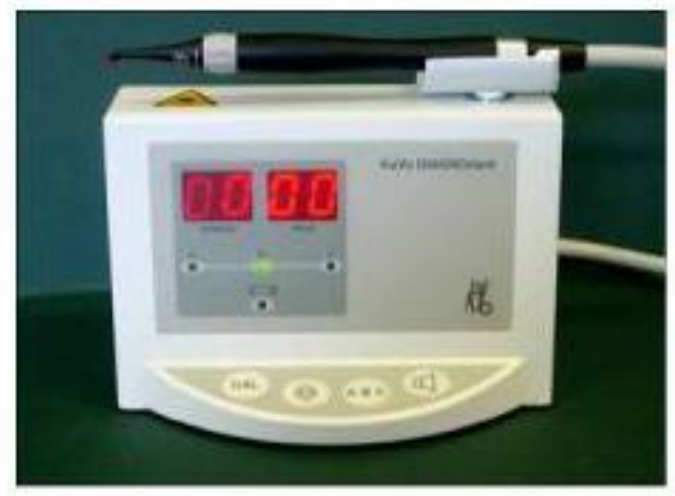

Fig no 4: Diagnodent
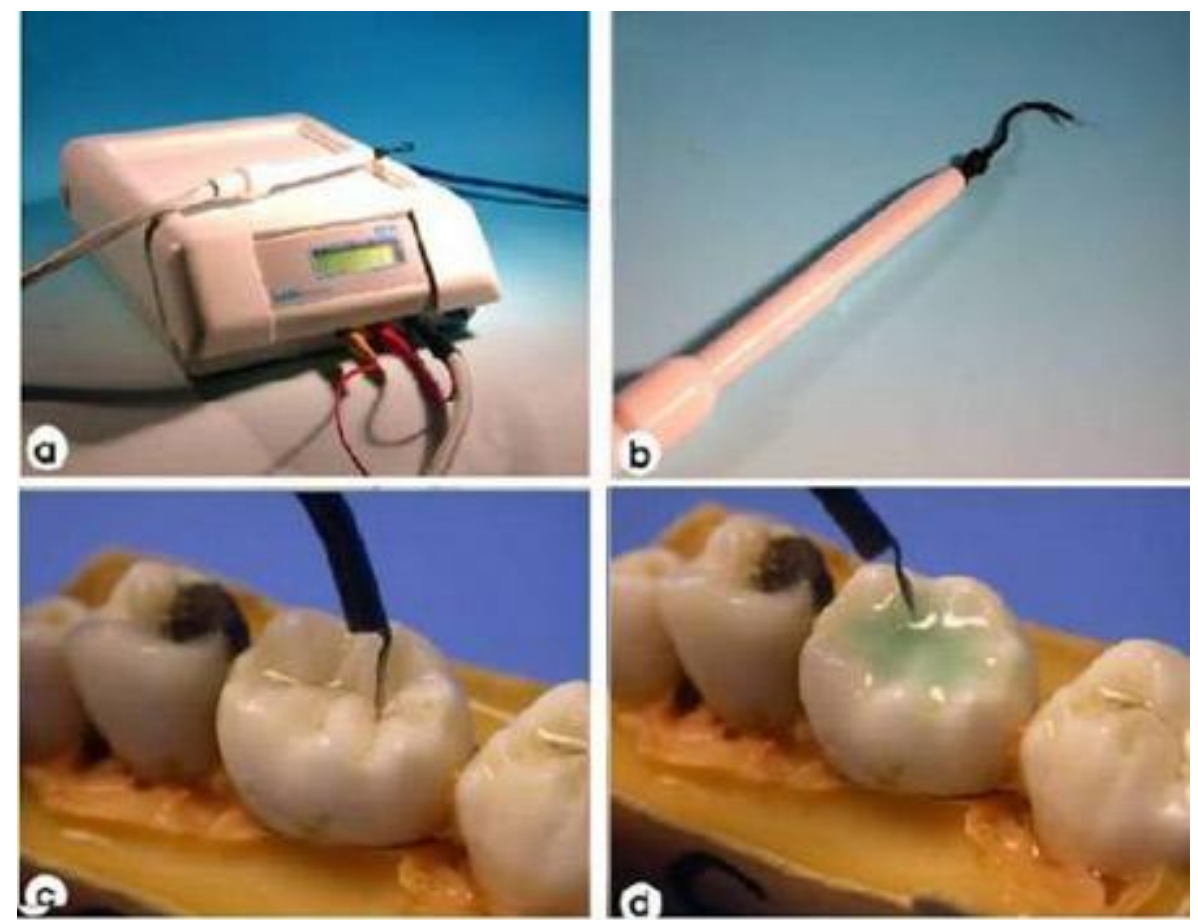

Fig 5: a) The ECM machine; b) the ECM handpiece;

c) site specific measurement technique; d) surface specific measurement technique 


\section{AUTHORS:}

1. Prachi Mital

2. Neha Mehta

3. Aditya Saini

4. Deepak Raisingani

5. Medhavi Sharma

\section{PARTICULARS OF CONTRIBUTORS:}

1. Second Year Post Graduate, Department of Conservative Dentistry and Endodontics, Mahatma Gandhi Dental College and Hospital, Jaipur.

2. Third Year Post Graduate, Department of Conservative Dentistry and Endodontics, Mahatma Gandhi Dental College and Hospital, Jaipur.

3. First Year Post Graduate, Department of Conservative Dentistry and Endodontics, Mahatma Gandhi Dental College and Hospital, Jaipur.
4. Professor and HOD, Department of Conservative Dentistry and Endodontics, Mahatma Gandhi Dental College and Hospital, Jaipur.

5. Sr. Lecturer, Department of Conservative Dentistry and Endodontics, Mahatma Gandhi Dental College and Hospital, Jaipur.

\section{NAME ADDRESS EMAIL ID OF THE CORRESPONDING AUTHOR:}

Dr. Prachi Mital, Post Graduate Second Year, Department of Conservative Dentistry and Endodontics,

Mahatma Gandhi Dental College and Hospital, Sitapura (Jaipur) - 302022.

Email-drprachi87@yahoo.com

Date of Submission: 01/12/2013.

Date of Peer Review: 02/12/2013.

Date of Acceptance: 20/12/2013.

Date of Publishing: 03/01/2014 